\title{
EFEITO DA ESCARIFICAÇÃO SOBRE ALGUNS PARÂMETROS FÍSICOS DE UM PLANOSSOLO(1)
}

\author{
R.L.T. MACHADO ${ }^{(2)}$, A.L. TURATTI ${ }^{(3)}$, A.S. ALONÇO ${ }^{(4)}$, \\ A.L.T. MACHADO ${ }^{(5)} \&$ A.V. REIS ${ }^{(5)}$
}

\begin{abstract}
RESUMO
Em face da importância do planossolo (Albaqualf) nas atividades agrícolas desenvolvidas na região Sul do estado do Rio Grande do Sul, Brasil, estudou-se o efeito de um escarificador sobre alguns parâmetros físicos desse solo, no Campo Experimental de Máquinas Agrícolas da E MBRAPA-CPACT, no Município do Capãodo-Leão, em novembro de 1994. Analisou-se esse efeito sobre a densidade, porosidade e agregação do solo, uti lizando três velocidades de operação do escarificador (4,76; 3,63 e $\left.2,58 \mathrm{~km} \mathrm{~h}^{-1}\right)$ e dois teores de água $\left(119,6\right.$ e $\left.71,7 \mathrm{~g} \mathrm{~kg}^{-1}\right)$ no solo. Não houve efeito significativo da interação teor de água $x$ velocidade de operação sobre os parâmetros físicos do solo. As condições físicas do solo foram melhoradas, quando o solo foi preparado com maior teor de água no solo, com exceção da densidade e macroporosidade do solo, as quais não foram influenciadas pelo teor de água no solo. A velocidade de deslocamento do escarificador não apresentou influência sobre os parâmetros físicos do solo estudados, com exceção do diâmetro médio geométrico e estabilidade dos agregados do solo, que apresentaram melhores resultados na menor velocidade de deslocamento.
\end{abstract}

Termos de indexação: propriedades físicas, umidade do solo, velocidade de operação do escarificador, planossolo.

\section{SUMMARY: EFFECTS OF CHISELLING ON PHYSICAL PARAMETERS OF A PLANOSOL}

Facethepl anosols (Albaqualf) importanceto theagricultural activities in theSouth Region of the State of Rio Grande do Sul, Brazil, the effect of chiselling on physical parameters of this soil was studied. Experiments were carried out at the Agricultural Machines Experimental Area of EMBRAPA - CPACT, in Capão do Leão, RS, November, 1994. It was analysed the effect of chisel ling at threeoperating speeds $\left(4.76 ; 3.63\right.$ and $\left.2.58 \mathrm{~km} \mathrm{~h}^{-1}\right)$, in two level s of soil moi sture

\footnotetext{
(1) Parte da tese do primeiro autor para a obtenção do título de Mestre em Agronomia pela Universidade Federal de Pelotas (RS). Recebido para publicação em maio e aprovado em agosto de 1997.

(2) Prof. Substituto, Mestre. Departamento de Solos, Faculdade de Agronomia Eliseu Maciel, UF Pel, Caixa Postal 354. CEP $96001-970$ Pelotas (RS), Brasil. Fone (0532) 757267. Fax (0532) 759031. (E-mail: rlilles@ufpel.tche.br)

(3) Professor Adjunto do Departamento de Solos da Faculdade de Agronomia Eliseu Maciel da Universidade Federal de Pelotas (RS). Caixa Postal 354. CEP 96001-970 Pelotas (RS).

(4) Pesquisador, Mestre, Centro de Pesquisa Agropecuária de Clima Temperado, EMBRAPA, Caixa Postal 403. CEP 96001-970 Pelotas (RS).

(5) Professor Assistente do Departamento de Engenharia Rural da Faculdade de Agronomia Eliseu Maciel da Universidade Federal de Pelotas (RS). Caixa Postal 354. CEP 96010-900 Pelotas (RS).
} 
content (119.6 and $71.7 \mathrm{~g} \mathrm{~kg}^{-1}$ ), on bulk density, porosity and soil aggregation. No significant effects due to interaction between moi sturecontent and operating speed were observed. Except for bulk density and macroporosity, which werenot affected by water content, physical conditions were improved when the soil was prepared with the highest moisture level. Geometric mean diameter and aggregatestability showed the best results at the lowest operating speed, but no effects of chisel speed were observed on other physical parameters.

Index terms: physical properties, soil moisture, chisel operating speed, planosol .

\section{INTRODUÇÃO}

Os sol os de várzeas do Sul do Brasil compreendem aqueles que, a despeito de grandes variações, apresentam uma característica comum: formação em condições de hidromorfismo (Gomes et al., 1992).

Os principais problemas enfrentados na viabilização do estabel ecimento das culturas de sequeiro, nas áreas de várzeas, estão relacionados com os atributos físicos do solo. Dentre esses, podem ser citadas alta densidade, baixa porosidade, alta relação micro/macroporos, baixa capacidadedearmazenamento deágua, condutividade hidráulica reduzida e baixa vel ocidade de infiltração de água no solo.

E $m$ determinações realizadas em unidades de mapeamento de solos hidromórficos do Rio Grande doSul, foram encontradas densidades do sol o superiores a 1,6 kg dm-3, nos horizontes superficiais (Silveira et al., 1989). A densidade em planossolos varia de 1,5 a $1,6 \mathrm{~kg} \mathrm{dm}^{-3}$.

A densidade do sol oé uma característica física que sofreal terações com o manejo, sendo um dos principais parâmetros físicos utilizados para avaliar a compactação do solo.

Os solos de várzeas apresentam alguns aspectos favoráveis à agricultura, tais como: baixa suscetibilidade à erosão, condições favoráveis à irrigação e facilidade de uso de máquinas agrícolas devido ao relevo plano.

O uso intenso da mecanização provoca alterações na estrutura do solo, proporcionando condições desfavoráveis ao crescimento das plantas, decorrentes da formação decamadas compactadas que provocam uma redução do volume de poros, aumentando, assim, a densidade do solo (Machado et al., 1981; N ovak et al., 1992).

Por localizar-se essa camada compactada próxima à superfície, há necessidade de seu rompimento, daí o uso de escarificadores e/ou subsoladores. Todavia, para o uso eficiente desses equi pamentos, é de fundamental importância que sejam analisados os parâmetros relativos à mobilização do solo e desempenho do equipamento.

A estrutura do solo, que é al terada pelo preparo, é avaliada pela determinação do grau de agregação, da estabilidade dos agregados e da natureza do espaço dos poros.

Mais importante que uma simples consideração sobre a magnitude da porosidade total de um solo, com vistas em caracterizar suas propriedades, é conhecer a distribuição do tamanho de seus poros (Kiehl, 1979; Gomes et al., 1992) e, em especial, dos macroporos, que são aqueles que mais contribuem para a porosidade de aeração. Baver et al. (1972) mencionaram que a macroporosidade aumenta com a agregação do sol o e com o tamanho dos agregados.

A melhor distribuição do tamanho dos agregados estáveis em água indica boas características físicas do solo, que se traduzem em el evada permeabilidade, retenção de água, arejamento, penetração de raízes e mel hor aproveitamento de nutrientes (Grohmann \& Arruda, 1961).

Braunack \& M cphee (1991), estudando o efeito do teor de água no solo sobre o tamanho dos agregados no momento do preparo, demonstraram que solos preparados a bai xos teores deágua formam agregados menores.

A melhoria, ou manutenção das condições físicas dos solos, não é conseguida apenas por meio de práticas mecânicas de preparo do solo. No entanto, quando corretamente empregadas, as práticas mecânicas são importantes para propiciar condições adequadas ao estabel ecimento das culturas. Uma dessas práticas éa escarificação, quetem por objetivos: reduzir a densidade do solo e a resistência à penetração de raízes; aumentar a permeabilidade; diminuir o escorrimento superficial da água da chuva em áreas com dedive, e o encharcamento em terrenos planos (Dalla Rosa, 1981).

Dos fatores que podem influenciar a relação máquina-solo destacam-se a velocidade de deslocamento do implemento de preparo do solo e o teor de água no solo.

As condições de umidade do sol o são importantes nas operaç̃ões de preparo do solo, pois a variação do nível de umidade regula a intensidade com que se manifestam as forças físicas de coesão e adesão, que atuam no interior do solo.

Stafford (1981) verificou que, em altos níveis de umi dade do solo, existem compressão efluidez do solo à frente e ao lado da haste do implemento de preparo do solo.

O preparo realizado com teores de água excessivamente altos (consistência plástica) causa o amassamento e a compactação do solo, enquanto o preparo com teores de água excessivamente baixos (consistência dura) resulta em pulverização do solo (Hillel, 1980). 
Esteestudo pretende contribuir para a viabilização deculturas de sequei ro nos sol os de várzea, anal isando o efeito do trabalho do escarificador, utilizando três vel ocidades de deslocamento e dois teores de água no solo, sobre al guns parâmetros físicos do solo, a saber: densidade, porosidade e agregação.

\section{MATERIAL E MÉTODOS}

Este estudo foi desenvolvido no Campo Experimental de Máquinas Agrícolas da EMBRAPA-

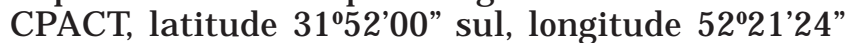
oeste, com altitude média de 13,24 m, localizado no município do Capão-do-Leão, Rio Grande do Sul, Brasil. O solo pertence à unidade de mapeamento Pelotas e, de acordo com IBGE (1986), é classificado, segundo o sistema brasileiro de classificação de solo, como um planossol o sol ódico, argila de atividade alta "A", moderado, textura média/argilosa, relevo plano, e como Albaqualf, segundo o sistema americano de classificação de sol o - 7ạ aproximação.

A análise granulométrica do solo (horizonte A), efetuada pelo método de Bouyoucos, forneceu os seguintes resultados: areia total $720 \mathrm{~g} \mathrm{~kg}^{-1}$; silte $174 \mathrm{~g} \mathrm{~kg}^{-1}$, argila $106 \mathrm{~g} \mathrm{~kg}^{-1}$, sendo classificado, de acordo com o triângul o textural, como um sol o francoarenoso (Kiehl, 1979).

A determinação dos Limites de Atterberg apresentou limite de plasticidade igual a $165 \mathrm{~g} \mathrm{~kg}^{-1} \mathrm{e}$ limite de liquidez igual $176 \mathrm{~g} \mathrm{~kg}^{-1}$.

No experimento, foi utilizado um escarificador montado, modelo comercial AS-5, fabricado pela Indústria de Máquinas Busse Ltda.(6), com controle de profundidade por rodas, chassi porta ferramentas em forma trapezoidal, três hastes verticais retas e ponteiras estreitas, distribuídas alternadamente, ou seja, haste central à frente das hastes laterais, com espaçamento entre hastes de 0,50 m e profundidade de trabalho de 0,20 m.

As determinações do teor de água no solo, da densidade e da porosidade do solo foram feitas conforme EMBRAPA (1979).

O tamanho dos agregados do sol o foi avaliado por meio do diâmetro médio geométrico (DMG). $O$ índice de estabilidade de agregados (EA) foi determinado conforme Kemper \& Chepil (1965), modificado pelo Laboratório de Física do Solo do Departamento de Solos da FAEM/UFPel. O procedimento adotado foi o de passar as amostras deformadas em peneira com 9,52 $\mathrm{mm}$ de malha, secar ao ar e peneirá-las em peneira de 4,76 mm demalha. Assim, determinaramse oDMG e oEA a partir de agregados que passaram pela peneira de $4,76 \mathrm{~mm}$ de malha, sendo classes de agregados estudadas (peneiramento em água): 4,762,$0 ; 2,0-1,0 ; 1,0-0,5 ; 0,5-0,25$ e $<0,25 \mathrm{~mm}$.

\footnotetext{
(6) A citação de marcas comerciais não indicam recomendação dos autores.
}

Foram coletadas três amostras por parcela com espaçamento de 5,5 m, na camada de 0-0,20 m, e entre as linhas de escarificação, após a passagem do escarificador.

Foi utilizado o delineamento experimental de blocos ao acaso, sendo efetuados tratamentos em dois teores médios de água $(\mathrm{Ta})$ no solo no momento da operação de escarificação, Ta1 = 119,6 g kg-1 (por irrigação) e Ta2 $=71,7 \mathrm{~g} \mathrm{~kg}^{-1}$, em três velocidades de trabal ho do escarificador (V), V1 $=4,76 \mathrm{~km} \mathrm{~h}^{-1}, \mathrm{~V} 2=$ $3,63 \mathrm{~km} \mathrm{~h}^{-1}$ e V3 $=2,58 \mathrm{~km} \mathrm{~h}^{-1}$ e com profundidade de trabal ho constante de $0,20 \mathrm{~m}$.

No delineamento estatístico, foram considerados, como variáveis independentes, o teor de água no solo no momento da escarificação e a velocidade de trabal ho do escarificador e, como variáveis dependentes, a porosidade total, a microporosi dade, a macroporosidade e a densidade do solo, bem como o diâmetro médio geométrico e a estabilidade de agregados.

Considerando as variáveis independentes, foram definidos seis tratamentos, com três repetições, totalizando dezoito parcelas.

As parcelas mediram 3,0 m de largura por 20,0 m de comprimento, sendo os 5,0 m iniciais reservados para aprofundamento do subsolador e estabilização dos aparel hos, ficando a parcela com um comprimento útil de 15,0 m.

\section{RESULTADOS E DISCUSSÃO}

A interação velocidade de trabal ho $x$ teor de água não foi significativa para todos os parâmetros físicos do sol o estudados, indicando que o efeito da vel ocidade de trabalho independe do teor de água no solo (Quadro 1).

Não houve diferença significativa entre os teores de água e as velocidades de trabal ho em relação aos valores médios da densidade do solo, o que pode ser explicado pelo alto teor de areia, visto que, em solos de textura grossa, as possibilidades de arranjo das partículas não são muito grandes.

Os valores médios do diâmetro médio geométrico (DMG) apresentaram diferença estatística significativa entre as vel ocidades $\mathrm{V} 3 \mathrm{eV} 2$, mas ambas não diferiram em relação $\mathrm{V}$ 1. Com rel ação aos teores de água no solo, os valores médios do DMG mostraram-se diferentes ao nível de significância de $5 \%$. Notou-se que o DMG aumentou com o aumento do teor de água no solo (Quadro 1).

Observou-seque, no sol o preparado com mai or teor de água, aumentou a percentagem de agregados de maior diâmetro, o que leva a concluir que o teor de água no solo no momento da escarificação influi no DMG dos agregados formados no solo, fato também observado por Braunack \& M cphee (1991) eAdam \& Erbach (1992). A presença de agregados maiores proporciona mel horia na condição estrutural do solo, visto que maior valor do DMG do solo indica mel hores 
características físicas do solo, que se refletem em aumento da permeabilidade, do armazenamento de água, do arejamento do sol o e da penetração de raízes.

A estabilidade de agregados (EA) do solo, em seus valores médios, apresentou difer ença estatística significativa entre os teores de água no solo, observando-se que esta cresceu com o aumento do teor deágua no sol o, o quetambém foi constatado por Adam \& Erbach (1992).

No interval o entre o limite inferior de friabilidade e o limite de plasticidade, os solos apresentam-se friáveis, condição considerada ótima para o trabal ho com implementos agrícol as. No entanto, como olimite inferior de friabilidade não é determinado, encontrando-se, teoricamente, próximo ao ponto de murcha permanente $\left( \pm 70 \mathrm{~g} \mathrm{~kg}^{-1}\right.$ para planossolo), toma-se esse ponto como o limite inferior de friabilidade (sol o seco). Assim, a friabilidade máxima do solo encontra-se aproximadamente, para o planossolo, entre 70 e $165 \mathrm{~g} \mathrm{~kg}^{-1}$, devendo o solo apresentar agregação quando sob trabal ho mecânico.

Quanto mais próximo o teor de água no solo do limiteinferior de friabilidade, condição do menor teor de água no solo (Ta2), mais seco está o solo, então o manuseio mecânico mais o pulverizará.

Verifica-se que comTal obteve-se mel hor estrutura do solo, já que a estabi lidade dos agregados refere-se à resistência que os agregados do solo apresentam a influência desintegradora da água e do manuseio mecânico.

Considerando as velocidades de trabalho, EA apresentou diferença estatística significativa entreV3 eV2, porém não diferiram em relação a V1. Esse efeito significativo da velocidade de trabal ho podeser explicado pela mudança do ti po de ruptura dosol o (ruptura em planos de cizalhamento para ruptura escoada), causada por uma haste rígida (Stafford, 1981).

Observa-se que a microporosi dade do sol o diminui à medida que o teor de água no solo decresce, apresentando diferença estatística significativa (Quadro 1). Não houve diferença significativa entre os teores de água no solo e entre as velocidades de trabal ho em relação aos valores médios da macroporosidade do solo.

Quadro 1. Densidade do solo, diâmetro médio geométrico (DMG), estabilidade de agregados (EA), microporosidade, macroporosidade e porosidade total do solo nos três níveis de velocidade e dois níveis de teor de água no solo

\begin{tabular}{|c|c|c|c|c|}
\hline \multirow{2}{*}{$\begin{array}{c}\text { Teor de água } \\
\text { no solo }\end{array}$} & \multicolumn{3}{|c|}{ Velocidade } & \multirow{2}{*}{ Média } \\
\hline & 4,76 & 3,63 & 2,58 & \\
\hline \multirow[t]{2}{*}{$\mathrm{g} \mathrm{kg}^{-1}$} & \multicolumn{4}{|c|}{$-\mathrm{km} \mathrm{h}^{-1}$} \\
\hline & \multicolumn{4}{|c|}{ Densidade do solo ( $\left.\mathrm{kg} \mathrm{dm}^{-3}\right)$} \\
\hline $\begin{array}{r}119,6 \\
71,7\end{array}$ & $\begin{array}{l}1,59 \\
1,61\end{array}$ & $\begin{array}{l}1,54 \\
1,65\end{array}$ & $\begin{array}{l}1,53 \\
1,54\end{array}$ & $\begin{array}{l}1,55 a \\
1,60 a\end{array}$ \\
\hline \multirow[t]{2}{*}{ Média } & $1,60 A^{*}$ & $1,60 \mathrm{~A}$ & $1,54 \mathrm{~A}$ & \\
\hline & \multicolumn{4}{|c|}{ DMG (mm) } \\
\hline $\begin{array}{r}119,6 \\
71,7\end{array}$ & $\begin{array}{l}0,78 \\
0,73\end{array}$ & $\begin{array}{l}0,76 \\
0,70\end{array}$ & $\begin{array}{l}0,80 \\
0,74\end{array}$ & $\begin{array}{l}0,78 a \\
0,72 b\end{array}$ \\
\hline \multirow[t]{2}{*}{ Média } & $0,75 \mathrm{AB}$ & $0,73 B$ & $0,77 \mathrm{~A}$ & \\
\hline & \multicolumn{4}{|c|}{ E A do solo (\%) } \\
\hline $\begin{array}{r}119,6 \\
71,7\end{array}$ & $\begin{array}{l}74,05 \\
58,04\end{array}$ & $\begin{array}{l}71,90 \\
56,31\end{array}$ & $\begin{array}{l}76,28 \\
63,24\end{array}$ & $\begin{array}{l}74,08 a \\
59,20 b\end{array}$ \\
\hline \multirow[t]{2}{*}{ Média } & $66,05 A B$ & $64,11 B$ & $69,76 A$ & \\
\hline & \multicolumn{4}{|c|}{ Microporosidade do solo $\left(\mathrm{m}^{3} \mathrm{~m}^{-3}\right)$} \\
\hline $\begin{array}{r}119,6 \\
71,7\end{array}$ & $\begin{array}{l}0,30 \\
0,24\end{array}$ & $\begin{array}{l}0,29 \\
0,26\end{array}$ & $\begin{array}{l}0,29 \\
0,27\end{array}$ & $\begin{array}{l}0,29 a \\
0,26 b\end{array}$ \\
\hline \multirow[t]{2}{*}{ Média } & $0,27 \mathrm{~A}$ & $0,27 \mathrm{~A}$ & $0,28^{A}$ & \\
\hline & \multicolumn{4}{|c|}{ Macroporosidade do solo $\left(\mathrm{m}^{3} \mathrm{~m}^{-3}\right)$} \\
\hline $\begin{array}{r}119,6 \\
71,7\end{array}$ & $\begin{array}{l}0,08 \\
0,08\end{array}$ & $\begin{array}{l}0,10 \\
0,07\end{array}$ & $\begin{array}{l}0,09 \\
0,08\end{array}$ & $\begin{array}{l}0,09 a \\
0,08 a\end{array}$ \\
\hline \multirow[t]{2}{*}{ Média } & $0,08 \mathrm{~A}$ & $0,09 A$ & $0,08 \mathrm{~A}$ & \\
\hline & \multicolumn{4}{|c|}{ Porosidade total do solo $\left(\mathrm{m}^{3} \mathbf{~ m}^{-3}\right)$} \\
\hline $\begin{array}{r}119,6 \\
71,7\end{array}$ & $\begin{array}{l}0,38 \\
0,33\end{array}$ & $\begin{array}{l}0,39 \\
0,34\end{array}$ & $\begin{array}{l}0,38 \\
0,36\end{array}$ & $\begin{array}{l}0,38 a \\
0,34 b\end{array}$ \\
\hline Média & $0,35 \mathrm{~A}$ & $0,36 \mathrm{~A}$ & $0,37 \mathrm{~A}$ & \\
\hline
\end{tabular}

\footnotetext{
* Médias seguidas da mesma letra (minúscula) na vertical e mesma letra (maiúscula) na horizontal não diferem significativamente
} entre si, ao nível de 5\%, pelo teste de Tukey. 
O val or médio da porosidade total do solo cresceu com o aumento do teor de água no solo, apresentando diferença estatística significativa entre os teores de água no solo. $\mathrm{O}$ aumento da porosidade total do solo, com o aumento do teor deágua no solo, já era esperado, pois tanto a microporosidade como a macroporosidade aumentaram com o teor de água.

O efeito do teor de água no solo, no momento da escarificação, sobre a microporosidade e porosidade total do solo é explicado pelo aumento do DMG dos agregados do solo com o aumento do teor de água, já que a porosidade do solo é alterada em função de mudanças no tamanho dos agregados (Larson, 1964 \& Baver et al., 1972).

Os maiores valores da EA, do DMG e da macroporosidade do solo, com o teor de água no solo Tal, evidenciam que, nessas condições, o solo se apresenta estruturalmente mais estável com mel horias nas suas condições físicas.

\section{CONCLUSÕES}

1. Na condição de maior teor de água no solo (119,6 g kg-1 ), a escarificação mostrou melhores resultados para os parâmetros físi cos estudados, com exceção da densidade e macroporosidade do sol o, que não foram influenciadas pelo teor de água no solo.

2. A velocidade de deslocamento do escarificador não apresentou influência sobre os parâmetros físicos do solo estudados, com exceção do diâmetro médio geométrico e estabilidade de agregados do solo, que apresentaram melhores resultados na menor velocidade de deslocamento do escarificador $\left(2,58 \mathrm{~km} \mathrm{~h}^{-1}\right)$.

\section{LITERATURA CITADA}

ADAM, K.M. \& ERBACH, D.C. Secondary tillage tool effect on soil aggregation. Trans. ASAE, St. J oseph, 35:1771-1776, 1992.

BAVER, L.D., GARDNER, W.H., GARDNER, W.R. Fisica de suelos. México, Hispano Americano, 1972. 529p.
BRAUNACK, M.V. \& MCPHEE, J.E. The effect of initial soil water content and tillage implement on seedbed formation. Soil Til. Res., Amsterdam, 20:5-17, 1991.

DALLA ROSA, A.D. Práticas mecânicas e culturas na recuperação de características físicas de solos degradados pelo cultivo solo Santo Angelo (Latossolo Roxo Distrófico). Porto Alegre, Universidade Federal do Rio Grande do Sul, 1981. 136p. (Dissertação de Mestrado)

EMBRAPA. Serviço Nacional de Levantamento e Conservação de Solos. Manual de métodos de análise de solo. Rio de J aneiro, EMBRAPA/SNLCS, 1979. n.p.

GOMES, A. da S., CUNHA da, N.G., PAULETTO, E.A., SI LVEIRA, R.J.C. da \& TURATTI, A.L. Solos de várzeas uso e manejo: problemas físicos e perspectivas de solução. In: MARCANTÔNIO, G. ed. Solos e irrigação. Porto Alegre, UFRGS/FEDERACITE, 1992. p.64-79.

GROHMANN, F. \& ARRUDA, H.V. Influência e preparo do solo sobre a estrutura da terra-roxa-legítima. Bragantia, Campinas, 20:1203-1209, 1961.

HILLEL, D. Fundamentals of soil physics. New York, Academic Press, 1980. 413p.

INSTITULO BRASILEIRO DE GEOGRAFIA E ESTATÍSTICA. Fol ha SH.22 Porto Alegre e parte das folhas $\mathrm{SH} .21$ Uruguaiana e SI.22 Lagoa Mirim: geologia, geomorfologia, vegetação, uso potencial da terra. Rio de J aneiro, 1986. 796p. (Levantamento de Recursos Naturais, 33)

KEMPER, W.D. \& CHEPIL, W.S. Size distribution of aggregates. In: BLACK, C.A. (ed.). Methods of soil analysis. Madison, American Society of Agronomy, 1965. v.1. p.499-506.

KIEHL,E.J. Manual de edafologia. São Paulo: Editora Agronômica Ceres Ltda., 1979. 262p.

LARSON, W.E. Soil parameters for evaluating tillage needs and operations. Soil Sc. Soc. Am. Proc., Madison, 28:118-122, 1964.

MACHADO, J.A., PAULA SOUZA, D.M. \& BRUM, A.C.R. Efeito de anos de cultivo convencional em propriedades físicas do solo. R. bras. Ci. Solo, Campinas, 5:187-189, 1981.

NOVAK, L.R., MANTOVANI, E.C., MARTYN, P.J . \& BAYRON, F. Efeito do tráfego de trator e da pressão de contato pneu/solo na compactação de um Latossolo Vermel ho-Escuro álico, em dois níveis deumidade. Pesq. agropec. bras., Brasília, 27:15871595, 1992.

SILVEIRA, R.J.C., GOMES, A. da S. \& PAULETTO, E.A. Levantamento de características químicas, físicas e mineralógicas dos principais sol os do RS. Pelotas, EMBRAPACPATB, 1989. n.p. v.1. 